\title{
Il codice Amadei IV della Universitaria di Bologna.*
}

Con la designazione di Amadei IV intendo di indicare quella sezione del cod. di rime antiche italiane della Universitaria I 289 che è costituita dalle cc. $97-134$, le quali formano un tutto indipendente dalle restanti parti del predetto manoscritto.

Il ms. 1289, il primo dei quattro che costituiscono il codice Amadei, 1 risulta di parecchie sezioni, dovute tutte a diverse mani del sec. XVI: I (cc. I-48); II (cc. 49-96); III (cc. 94-96); IV (cc. 97-1 134); $\nabla$ (cc. 135-139); VI (cc. 148-158, le cc. 140-147 sono bianche); vII (cc. 159-1 74); vIII (cc. 175-213).

Queste varie sezioni, un tempo indipendenti, com' è dimostrato da una vecchia numerazione, furono poi accostate l' una all' altra, e rilegate insieme formarono il nostro cod. 1289 , da molti consultato, da tutti gli studiosi delle nostre lettere assai conosciuto, ma non ancora esaminato con la diligenza che merita. Tuttavia per la prima sezione (Amadei I), siamo meglio informati che per l'altre, in quanto sappiamo ch' essa è depositaria, col vatic. 3214, di gran parte dei componimenti di un celebre cod. perduto, posseduto dal Beccadelli.1 Mi accingo ora io stesso all' esame della sezione IV, fiducioso di giovare qualche poco agli studi, appena iniziati, sulla costituzione dei nostri antichi canzonieri. Tuttavia il lettore non isdegnerd qualche nota intorno ad alcune particolarità del ms. 1289 e degli altri. Una parte (B) del cod. Amadei è tutta di mano di G. M. Barbieri; ${ }^{2}$ le cc. $94-96$ del cod. 1289

* Alcane poche parole sui criteri seguiti in questo mio studiolo. Dimostrata l'atfinità strettissima fra il cod. Amadei IV e il ms. casanatense d. v. 5 (affinità che era stata già veduta, se non ancor dimostrata, da altri, per es. da A. F. Massèra, I sonetti di Cecco Angiolieri, Bologna, Zanichelli, 1905, p. XXXIII sgg.), era inutile del tutto ch' io riproducessi per intero il ms. Amadei IV. Ne ho rilevate invece tutte le varianti, persino quelle grafiche, tenendo sott' occhio la stampa del casanatense data dal Pelaez.

1 A. F. Massera, Su la genesi della raccolta Bartoliniana, in Zeitschrift f. roman. Philol., XXVI, I sgg.

2 Il canonico G. G. Amadei possedette quattro codd. di rime antiche. ch' egli numerò progressivamente in verzino, e che nel loro complesso furon chiamati dal Quadrio "il codice Amadei". La frase, o meglio la designazione, ha fatto fortuna: e $\mathrm{i}$ codd. ritrovati per le ricerche del Lamma, Giorn. stor. d. lett. ital., XX, 15 I e del Frati, L., Giorn., cit., XXIV, 300 sono i seguenti: A (Codice 1289); B (7118), C (1072'1), D (401).

Zeitschr, f rom. Phil XXX. 
(A, III), contenenti una corrispondenza poetica, in assai buona lezione, fra Cecco di Meletto de' Rossi da Forli, il Petrarca, l' Anguissoli, il Beccari e il Boccaccio, son dovute alla stessa mano che scrisse l' indice degli autori nel cod. bolognese 2448 e vi premise una notizia sulla sua derivazione, e le postille che si trovano nella prima sezione del 1289 sono indubbiamente di chi postillo qua e là il ms. 2448 e vi copiò in fine da un „libro Strozzi" alcuni componimenti. Fatti gli opportuni riscontri con l' aiuto anche della perizia altrui, credo di poter ciò affermare con sicurezza.2 Uno stesso erudito del sec. XVI ebbe dunque tra mano: I. la prima sezione del cod. Amadei; 2. il cod. 2448 ; 3 . il cod. Strozzi, che è ora l' Ashb. 763 .

Chi ponga gli occhi sul cod. Amadei IV, noterà facilmente la sua affinità con due altri manoscritti di rime antiche italiane: il cod. casanatense d. v. 5 e il così detto ms. Galvani.

Il cod. casanatense, pubblicato di recente, ${ }^{3}$ è cartaceo del sec. XVI, ed è stato descritto brevemente dal Monaci4 dal Mazzatinti ${ }^{5}$ e con maggior larghezza da M. Pelaez. ${ }^{6} \mathrm{Mi}$ accontento percio di rimandare a quest' ultima descrizione, e mi fermo invece qualche poco sul ms. Galvani.

Gio. Galvani cita, com' è noto, più d' una volta un suo codicetto di rime antiche donatogli dal Signor De Latur. Lo ricorda nel Saggio di alcune postille alla divina Commedia, Modena, 1828, p. 39, nelle sue Osservazioni sulla poesia de' trovator, Modena, 1829, p. 46 e infine nelle Lezioni accademiche, Modena, 1840, II, I 20. Il codicetto consisteva di 28 carte, unite ad un esemplare della Giuntina, postillato dalla stessa mano, e rilegate in fine. Era dell' a. 1547. Morto il Galvani, il manoscritto passò al libraio bolognese Carlo Ramazzotti, dal quale l' ebbe il co. Giacomo Manzoni. Presso il Manzoni lo conobbe il Casini, Le rime dei poeti bolognesi del sec. XIII, Bologna, I881, p. XVIII, che ebbe copia, per mezzo di C. Ricci, di otto componimenti, di cui diede le varianti:

1. Madonna, il fino amore ch' eo ve porto (p. 18).

2. $O$ falso amor, che credi di me fare (p. 91).

3. Amico, dir te voi' questo cotanto (p. 92).

1 Ho gia avuto occasione di assodar cio prima in un mio articolo $I$ codd. di rime antiche del Barbieri, in Giorn. stor. della lett. ital., XL, $35 \mathrm{sgg.} \mathrm{e}$ poscia nel mio volume Gio. Maria Barbieri e gli studi romanzi nel sec. XVI, Modena, 1905, p. 100.

- Circa le postille del cod. Amadei 1289 ho dovuto, esaminando bene attentamente le cose, abbandonare il sospetto che possano ascriversi al Barbieri, al quale appartiene senza fallo $\mathrm{l}^{\prime}$ intero cod. $7 \mathrm{I}^{3}$.

- M. Pelaez, Rime ant. ital. secondo la lexione del cod. vatic. 3214 e del cod. casanatense d. $v$. 5, in Collez. di op. ined. o rare, Bologna, 1895.

- Rime e lettere di ser Ventura Monaci, Bologna, 1879, p. 16.

- Studi di flol. rom., I, p. 330.

- Op. cit., p. XV sgg. 
4. Mente et umile e più di mille sporte (p. 93).

5. Amor che vien per le piu dolci parte (p. 94).

6. Non po' gioir d'amor chi non pareggia (p. 142).

7. Dolce d'amore amico, eo ve riscrivo (p. 143).

8. Poi ch' il pianeto ve dà fe' certana (p. 144).

Ora il manoscritto è andato perduto, ma se ne ha la tavola, tratta dalle carte del Bilancioni, edita per cura del Barbi, Studi di mss. e testi inediti, Bologna, Zanichelli, I900, p. 70. È lecito sperare che un giorno o l'altro il nostro codicetto ritorni alla luce; ma per ora dovranno gli eruditi tenersi contenti a quel poco che ne hanno dato il Casini e il Barbi?

Tra le carte del Co. Gio. Galvani, conservate nell' autografoteca Campori, si rinviene una descrizione incompleta del codice dovuta allo stesso Galvani, il quale s' è industriato di rilevarne l' importanza per mezzo dell' esame delle varianti di parecchi componimenti. Riporto in appendice quel tanto che può essere d' utilità agli studi sulla lirica delle nostre origini.1 Qui mi basterà avvertire che i punti di contatto fra il cod. Galvani e il casanatense sono molti e singolari, come apparirà dal seguente specchietto:

\begin{tabular}{|c|c|c|c|c|c|c|c|c|c|c|}
\hline $\begin{array}{r}1 \\
67\end{array}$ & $\begin{array}{r}2 \\
140\end{array}$ & $\begin{array}{r}3 \\
73\end{array}$ & $\begin{array}{r}4 \\
74\end{array}$ & $\begin{array}{r}5 \\
76\end{array}$ & $\begin{array}{r}6 \\
77 \\
\end{array}$ & $\begin{array}{r}7 \\
78\end{array}$ & $\begin{array}{r}8 \\
80\end{array}$ & $\begin{array}{r}9 \\
82\end{array}$ & $\begin{array}{r}10 \\
83\end{array}$ & $\begin{array}{l}11 \\
87\end{array}$ \\
\hline 12 & 13 & 14 & 15 & 16 & 17 & 18 & 19 & 20 & 21 & 22 \\
\hline 84 & 85 & 86 & 141 & 188 & 91 & 95 & 98 & 99 & 100 & 68 \\
\hline 23 & 24 & 25 & 26 & 27 & 28 & 29 & 30 & 31 & 32 & 33 \\
\hline 110 & 104 & 114 & 238 & 117 & 118 & II & 121 & 122 & 123 & I 39 \\
\hline $\begin{array}{r}34 \\
125\end{array}$ & $\begin{array}{r}35 \\
127\end{array}$ & $\begin{array}{r}36 \\
129\end{array}$ & $\begin{array}{r}37 \\
131\end{array}$ & $\begin{array}{r}38 \\
106\end{array}$ & $\begin{array}{r}39 \\
103\end{array}$ & $\begin{array}{r}40 \\
108\end{array}$ & $\begin{array}{r}41 \\
159\end{array}$ & $\begin{array}{r}42 \\
168\end{array}$ & $\begin{array}{r}43 \\
163\end{array}$ & \\
\hline
\end{tabular}

1 Tra le carte del Bilancioni (cfr. L. e C. Frati, Indice delle carte di P. Bilancioni, Bologna, 1893, p. 9 n. 1), nella Civica di Bologna, deve esservi copia di tutto il ms. Io vi trovo pure:

10. Una lettera di Bartolomeo Veratti (14. II. 1865) con la quale egli spedisce al Bilancioni la tavola, fatta dal Conte Galvani, del codice in discorso.

20. La tavola di mano del Co. Galvani con la seg. descrizione: „Il Codicetto Galvani è di cc. 28 del formato stesso della raccolta di rime antiche pubblicate in Firenze dai Giunta nel 1527, e rilegato dietro la medesima. Porta la data 1547." E in fine: "Sui margini poi dell' edizione Giuntina sono apposte numerose varianti che si riscontrano uscite dalla stessa mano che scrisse il codicetto aggiunto."

$3^{\circ}$. Un confronto tra il contenuto del cod. Galvani e il cod. Casanatense D. V. 5 .

$4^{\circ}$. Una lettera di Ant. Cappelli (19 Maggio 1868), nella quale si tocca della stampa del Cappelli, Otto sonetti del sec. $X I V$, Modena, 1858, (Nozze Della Volpe-Zambrini).

Trovasi pure, tra i componimenti dei vari poeti, la copia di quelli del cod. Galvani; ma parecchie cose cred' io che manchino. 


$$
\begin{aligned}
& \begin{array}{lrr||rrrr||r||r||r||rr}
\text { G. } & 45 & 46 & 47 & 48 & 49 & 50 & 51 & 52 & 53 & 54 & 55 \\
\text { C. } & 144 & 145 & 137 & - & - & 175 & 147 & - & - & 160 & -
\end{array}
\end{aligned}
$$

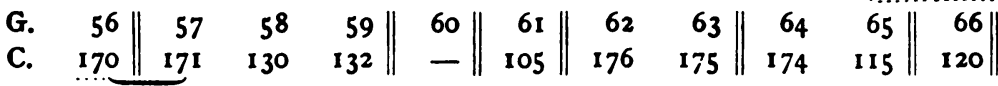

$$
\begin{aligned}
& \begin{array}{lrrr||r||r||r||r}
\text { G. } & 67 & 68 & 69 & 70 & 71 & 72 & 73 \text { (anon.) } \\
\text { C. } & 124 & 126 & 128 & - & - & 143 & 173 \text { (anon.) }
\end{array}
\end{aligned}
$$

Mancano adunque al cod. casanatense i segg. componimenti del cod. Galvani:

No. 48. O falso amor che credi di me fare Onesto

" 49. Amico dir ti vo' questo cotanto $\}$ bolognese

"52. La luna e il sole sono pianeti boni, G. Orlandi.

" 53. Saturno e Marte stelle infortunate, G. Romitano.

$\eta$ 54. Se io avessi un sacco di forini, C. Angiolieri.

" 6o. Uomo saccente eे da maestro saggio, Mula de' Muli.

"70. A fine di riposo ho sempre affanno, Ben. Salimbeni.

"7 1. Mostraci il mondo prode e dacci danno, Bindo Bonichi.

I nni. 48-49 sono unici nel nel cod. Galvani; il $n^{0} .52$ trovasi nel Vat. 3214 , c. $145^{2}$; il 53 pure:

$$
\begin{array}{lrr}
\text { Vat. 3214 } & 123 & 122 \\
\text { Cod. Galv. } & 52 & 53
\end{array}
$$

il 60 in Bol. 1289 , c. $101^{2} ; 70$ in cod. Triv. 1058,71 pure in Triv.

Importanti sono pure le relazioni che intercedono tra il codice casanatense e il ms. Amadei IV e tra questo e il cod. Galvani. Si tratta di una strettissima parentela, come apparirà dal registro, che facciamo seguire, e dalla tavola delle concordanze:

$$
\begin{gathered}
\text { Cod. I 289, (Sez. IV). } \\
\text { (cc. } 98-158 .)
\end{gathered}
$$

I. c. $97^{2}$. Dante a ms. Cino, Io mi credea del tutto esser partito.

2. c. $97^{b}$. Risposta di ms. Cino a Dante, Poi ch' io fui Dante del mio natal sito.

3. c. $98^{2}$. Dante a $M$. Cino, Perch' io non trovo chi meco ragioni. Cod. Galv. 1.

4. c. $98^{\text {b }}$. Riposta di M. Cino, Dante, io non odo in quale albergo suoni, Galv. 22.

5. c. $99^{2}$. Riposta di M. Cino al sonetto di $M$. Honesto il quale e in questoa c. 6, Amor che vien per le più dolci porte, Galv. 26.

6. c. $99^{b}$. Cino essendo a Prato ribello di Pistoia, Lasso pensando alla destructa valle, Galv. 39 .

7. c. $100^{2}$. M. Cino essendo alle Sambuche in sul monumento della vaga sua, Io fui in sul alto e' $\mathbf{n}$ sul beato monte, Galv. 40.

8. c. $100^{\mathrm{b}}$. Riposta di M. Cino al seguente di M. Mula, Ser Mula tu te credi senno avere, Galv. 23. 
9. c. ror a. M. Mula de Muli a M. Cino, Homo saccente è da maestro saggio, Galv. 60.

10. c. ror b. M. Cino a M. Cecco d'Ascoli, Cecco io ti prego per virtù di quella, Galv. 24.

11. c. 102a Risposta di M. Cecco a M. Cino, Di ciascheduna mi mostra la guida, Galv. 6r.

12. c. $1_{02}{ }^{b}$. M. Honesto a $M$. Cino, Mente humile et più di mille sporte, Galv. 47.

13. c. $103^{\mathrm{a}}$. M. Cino per lo libro di Dante, In verità questo libel di Dante, Manca Galv.

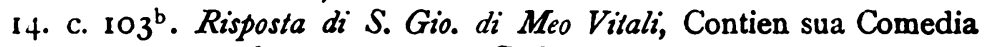
parole sante, manca Galv.

15. c. $104^{2}$. M. Cino sopra la detta materia, Infra gli altri difetti del libello, manca Galv.

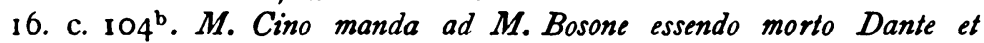
Emanuel Giudeo, M. Bosone lo vostro Manoello, Galv. 25 .

17. c. $105^{2}$. Risposta fatta in persona di M. Bosone, Manuel che mettesti in quello avello, Galv. 65.

18. c. 105 $5^{\mathrm{b}}$ M. Cino, Tutto ciò che altrui grada mi disgrada, Galv. 27 .

19. c. 106a. Id., Una ricca rocca et monte manto, Galv. 28 .

20. c. $106^{\mathrm{b}}$. Id. essendo a Perugia, Perchè voi state forse anchor pensivo, Galv. 29.

21. c. $107^{2}$. Gherardo da Reggio a M. Cino, Con sua saetta d' or percosse amore, Galv. 66.

22. c. $107^{\mathrm{b}}$. Risposta di M. Cino et consiglio sopra'l ditto sonetto, Amor che viene armato a doppio dardo, Galv. 30.

23. c. $108^{2}$. M. Cino, Quando ben penso il piccolino spatio, Galv. 31 .

24. c. $108^{\mathrm{b}}$. Id., Naturalmente ogni animale ha vita, Galv. 15 .

25. c. $109^{\mathrm{b}}$. Id. in essendo a Napoli, Dhe quando rivedrò il dolce paese, Galv. Io.

26. c. II $1^{2}$. M. Cino per lo imperadore Henrico di Lucimburgo quando mori, Da poi che la natura ha fine posto, Galv. II.

27. c. $113^{\mathrm{b}}$. M. Cino a M. Gherarduccio Garisendi da Bologna, Caro mio Gherarduccio io non ho veggia, Galv. 32.

28. c. II $4^{2}$. Risposta di $M$. Gherarduccio, non può zoir d' amor chi non pareggia, Galv. 67.

29. c. $114^{\mathrm{b}}$. Messer Cino a Dante, Cercando di trovar lumera in oro, Galv. 33 .

30. c. $115^{2}$. Risposta di Dante a M. Cino, Degno vi fe trovare ogni thesoro, Galv. 2.

31. c. $115^{\mathrm{b}}$. M. Cino a M. Gherarduccio, Amato Gherarduccio quando iscrivo, Galv. 34.

32. c. II $6^{\mathrm{a}}$. Risposta di $M$. Gherarduccio, Dolce d' amor amico, i' vi descrivo, Galv. 68.

33. c. $116^{\text {b }}$. M. Cino a $M$. Gherarduccio sopra la detta materia, Come li saggi di Neron crudele, Galv. 35. 
34. c. $117^{\mathrm{2}}$. Risposta di $M$. Gherarduccio a $M$. Cino, Poichè 'l pianeto vi dà fe certana, Galv. 69.

35. c. $117^{\mathrm{b}}$. M. Cino essendo a Pisa, al mio parer non è chi in Pisa porti, Galv. 36.

36. c. $118^{2}$. Risposta di Guelfo Taviani, Molto li tuoi pensier mi paion forti, Galv. 58 .

37. c. I $18^{\mathrm{b}}$. M. Cino, A la battaglia ove madonna abatte, Galv. 37 .

38. c. I1 $9^{2}$. Risposta di $M$. Guelfo, Pensando comei suoi sermoni adatte, Galv. 59.

39. c. $119^{b}$. M. Honesto da Bologna a M. Cino, Poscia che in cuor l' amorosa radice, manca Galv.

40. c. $120^{2}$. Risposta di M. Cino a M. Honesta bolognese, Anzi ch' amore ne la mente guidi, manca Galv.

41. c. $120^{b}$. M. Honesto a M. Cino, Assai son certo che sementa in lidi, Galv. manca.

42. c. $121^{2}$. Risposta di $M$. Cino a $M$. Honesto, Se mai legiesti versi delli Ovidi, manca Galv.

43. c. $121^{b}$. Canzon di M. Cino, Mille volte richiamo il dì mercede, manca Galv.

44. c. $123^{2}$. M. Cino, Lo sottil ladro che ne gli occhi porti, Galv. 39.

45. c. $124^{2}$. Canzone di $M$. Cino, Non che im presentia della vista humana, Galv. manca.

46. c. $125^{\text {b }}$. Zampa Ricciardi sopra la morte di $M$. Cino, Morto è colui ch' era archa della leggie, Galv. 72.

47. c. $126^{2}$. Sonetto di Manuel Giudeo, Amor non lesse mai l' avemaria, Galv. 62.

48. c. $12^{\mathrm{b}}$. Ceccho Angiellieri a Dante, Dante Alighier, Ceccho tuo servo e amico, Galv. 54 .

49. c. $127^{2}$. Il detto a Dante sopradetto, Dante Aleghier, s' io son bon begolardo, Galv. 56.

50. c. $127^{\mathrm{b}}$. M. Cane dalla Schala a Bologna, Ghelphi, el gran prence nobil de Stericcho, manca Galv.

51. c. 128a. M. Cino, Degno son io di morte, Galv. 4.

52. c. $130^{2}$. Id., Fior di virtù si è gentil corraggio, manca $\mathrm{G}$ alv.

53. c. $130^{b}$. Id., Io son sì vago della bella luce, manca Galv.

54. c. $130^{\text {bis. }}$. Id., Io guardo per li prati ogni fior biancho, Galv. manca.

55. c. $13^{\mathrm{b}}$. Id., Nouelle non di ueritade ignude, manca Galv.

56. c. $132^{2}$. Id. M. Cino, Oimè ch' io ueggio per entro un pensero, Galv. 17.

57. c. $132^{\mathrm{b}}$. Id., Quando potrò io dir dolce mio Dio, Gav. manca. 58. c. $133^{b}$. Del Petrarcha, Quella ghirlanda che la bella fronte, Galv. manca.

59. c. $134^{2}$. Id. Sostenne con le spalle Hercole il cielo, Galv. manca. 
Se indaghiamo a lor volta $i$ rapporti del cod. casanatense col ms. Amadei IV, troviamo le seguenti concordanze significative:

$\begin{array}{lrrrrrrrrrrr}\text { Am. } & 1 & 2 & 3 & 4 & 5 & 6 & 7 & 8 & 9 & 10 & 11 \\ \text { Cas. } & 65 & 66 & 67 & 68 & 138 & 103 & 108 & 110 & 109 & \underbrace{104} & 105 \\ \text { Am. } & 12 & 13 & 14 & 15 & 16 & 17 & 18 & 19 & 20 & 21 & 22 \\ \text { Cas. } & 137 & 111 & 112 & 113 & 114 & 115 & 117 & 118 & 99 & 120 & 121 \\ \text { Am. } & 23 & 24 & 25 & 26 & 27 & 28 & 29 & 30 & 31 & 32 & 33 \\ \text { Cas. } & 122 & 141 & 83 & 87 & 123 & 124 & 199 & 141 & 125 & 126 & 127 \\ \text { Am. } & 34 & 35 & 36 & 37 & 38 & 39 & 40 & 41 & 42 & 43 & 44 \\ \text { Cas. } & 128 & 129 & 130 & 131 & 132 & 133 & 134 & 135 & 136 & 85 & 106 \\ \text { Am. } & 45 & 46 & 47 & 48 & 49 & 50 & 51 & 52 & 53 & 54 & 55 \\ \text { Cas. } & 86 & 143 & 176 & 169 & 170 & 178 & 74 & - & 61 & - & - \\ \text { Am. } & 56 & 57 & 58 & 59 & & & & & & & \\ \text { Cas. } & 91 & 84 & - & - & & & & & & \end{array}$

Quanto alla disposizione adunque dei componimenti, il cod. Amadei ha maggiore attinenze col ms. casanatense che con quello Galvani. Ma che il cod. casanatense e il cod. Amadei IV non siano l' uno copia dell' altro è dimostrato da più fatti: per es. nel sonetto di messer Gherarducci, Dolce d' amore amico, il casanatenze legge l' ultimo verso così (ediz. cit., p. 307):

il cod. Galvani:

$$
\text { Cosi ui uolga d' ella ..... inta }
$$

$$
\text { Cosi ui nolga d' ella ..... cinta }
$$

il ms. Amadei IV:

Cosi ui uolga d' ella parum cinta

E chiaro che il copista di Amadei IV si è sforzato con l' incomprensibile purum di riprodurre la parola dell' originale saltata, perchè inintelligibile, dall' amanuense del casanatense e dall' amanuense del cod. Galvani.

E poi si confronti, per restarne appieno convinti, la lezione della strofe Il della canzone di Cino, Naturalmente ogni animale ha uita:

Casanatense, $99^{\mathrm{b}}$.

Amore che 'namora altrui per pregio

Lo qual a dio pareggia

Ch' è uertù somma che nasce ne [l' almo

Et quelli che s'adobba dil suo fregio

Et stimola et inueggia

- - - - almo

- - - - - affetto

Nel mondo, ne diletto,

Ch' hauer il suon de la uerace fama
Amadei.

Amore che iunamora altrui per pregio De uirtù somma che nasce ne l'almo La qual a dio pareggia

Et quelli che s' addobbu del suo fregio Et stimola et inueggia

Et opera diuerso et uaio Et affetto nel mondo ne' diletto Che hauere el suon de la nerace [fama 


\section{- - - - - - etto \\ Però chi non la brama}

Sta, come nel giardin la secca rama.
Po (l. perd) chi non la brama

Sta come nel giardin la secca rama.

Nella stanza III della canz. di Cino, Degno son io di morte, l' amanuense del cod. casanatense dimenticò il verso Piacciaui perdonare ch' è dato dal cod. Amadei. Il copista del qual manoscritto si attenne fedelmente all' originale anche quando non capiva. Uno studioso del sec. XVI, servendosi d' un altro ms., segnò qua e là alcune varianti di sua mano, p. es. nel componimento $n^{0} .2$ le fece scrivere dallo stesso copista, p. es. nella canzone ricordata Degno son io di morte, il cui ultimo verso fu modificato così: Usa nel tempo ch' ei pud gastigare, mentre il cod. casanatense dice: Usa nel tempo che si puo venghiare, alla qual lezione si riattaccava la prima scrittura del cod. amadei. Per tutte queste ragioni, e per altro ancora, non andremo dunque lontani dal vero, presentando lo schema seguente, come risultato del nostro studio:

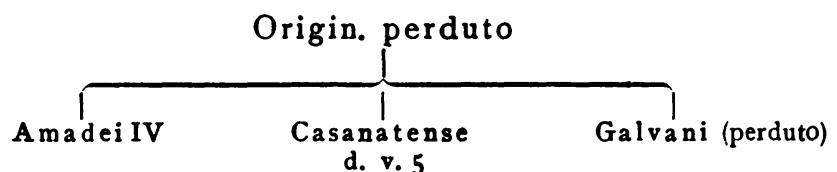

Varianti del codice Amadei IV rilevate sul casanatense d. v. 5. (c. 97a) Dante a M. Cino.

I. I mi credea del tutto esser partito; 2 rhime, 3 conuiene, 4 longi, 9 si come, II leggiermente, 13 uirtu, 14 il fatto.

(c. 97b) Risposta di M. Cino a Dante.

2. Poi ch' io fui Dante del mio natal sito

Facto per greve exilio peregrino

Et lontanato dal piacer più fino

Che mai formasse el sauere infinito.

Io son piangendo per lo mondo gito. isdegnato d'amor'

Ad guisa di tapin como mischino

$E$ sho trouato allui simil uecino

Detto ho che quelli $m$ 'ha lo

Ho detto che d'mha el cor ferito

Ne delle prime braccia di pictate,

Doue m 'accolue

Onde el fermato dispar mi solue perch' aita

Son mosso anchor che aiuto non aspetti.

1 Tra rigo e rigo segno le varianti dovute ad altra mano. 
Ch' un piacer

Un pensier sempre mi lega et inuolue

Di che conuien che in simil di beltate parti donne me

In molte donne sparte se diletti.

(c. 982) Dante a M. Cino.

3. Perch'io non trouo chi meco ragioni, 3 conuiemmi sasfure il, 4 de dire, 5 Nulla a., 7 che è, 9 li uenga, 10 lui, 12 Ahi.

(c. 98b) Risposta di M. Cino a Dante.

4. Dante io non odo in quale albergo suoni, 2 ciaschun, $3 E, 6$ fio 9 Donqua, II Uomi, $14 \mathrm{De}$.

(c. 99a) Risposta di M. Cino al sonetto di M. Honesto il quale è in questo a c. 6.

5. Amor che vien per le più dolci porte, 2 trappannando, 3 tien, 8 sb' iecta (subiecta), 13 Parlian, 14 di.

(c. $\left.99^{b}\right)$ Cino essendo a Prato ribello di Pistoia.

6. Lasso pensando alla destructa valle, 3 duole, 7 uuole, $8 \mathrm{Se} .$. del, Io dilecto, 13 Donque, 14 del.

(c. 100a) M. Cino essendo alle Sambuche in sul monumento della vaga sua.

7. Io fui in sul alto $e^{\prime} n$ sul beato monte, 2 Che a., 3 in, 5 uirtu, 8 addornezse, 9 ad, 11 ad.

(c. Ioob) Risposta di M. Cino al seguente di M. Mula.

8. Ser Mula tu te credi senno avere, 2 uirtí, 3 comune, 4 con, 9 inganno, 12 chella trouasti, 13 sext' anno, 14 cottal.

(c. 101a) M. Mula de Muli a M. Cino.

9. Homo saccente d da maestro saggio, 6 uien, 12 insegnato, 14 torno.

(c. 101b) M. Cino a M. Cecco d'Ascoli.

10. Cecco io ti prego per uirtì di quella, 4 in, $5 \mathrm{mi}, 8$ iudicio, 14 troue.

(c. 102a) Risposta di M. Cecco a M. Cino.

11. Di ciascheduna mi mostra la guida, 3 sexta, $5 E l \ldots$ et, 6 Ella 7 Del, 9 di.

(c. $102^{b}$ ) M. Honesto a M. Cino.

12. Mente humile et più di mille sporte, 3 rhimando, 9 pare, 10 parlare, 12 huomo, 13 donque, 14 siete.

(c. 1032) M. Cino per lo libro di Dante.

13. In ueritd questo libel di Dante, Mancano anche nel cod. Amadei i vv. 5 -6 , 10 et longhi.

(c. 103b) Risposta di S. di Gio. di Meo Vitali.

14. Contien sua comedia parole sante, 2 ad quelle che contan gli, 3 auctor ... fucesti, 4 in ... uieti, 5 gli c. exempli furon p. costante, 7 luogo, 9 el $u$., 10 chi g., II parlo il diritto conio, 14 che li ardi. 


\section{(c. 104a) M. Cino sopra la detta materia.}

15. Infra gli altri difetti del libello, 5 raggionando, 8 Rinaldo Daniello, e a lato, della stessa mano: Arnaldo Dariello, 9 L' altro ... canto suo, 10 choro, 12 Et $q$. ... seno, 13 recognobbe, 14 congionse.

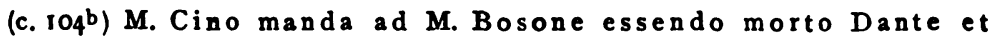
Emanuel Giudeo.

16. M. Bosone lo uostro Manoello, 4 se, 13 in.

(c. Io5a) Risposta fatta in persona di M. Bosone.

17. Manuel che metteti in quello auello, 2 che, 3 del ... ribbello, 4 seggie, 7 dipinto, 8 tali lau., 12 Emanuel compian, 13 medollo, 14 gionga.

$$
\text { (c. } \text { 105 }^{\mathrm{b}} \text { ) M. Cino. }
$$

18. Tutto cid che altrui grada mi disgrada, 3 donqua ... e ti, 4 aghiada, Io malenconia, II uorre, $13 \mathrm{ch}$ ' io, 14 io trono.

\section{(c. 106a) M. Cino.}

19. Una riccha roccha et monte manto, 4 ad ogni, 7 quine, 8 zoia, 12 fidele, 13 quei, 14 di

$$
\text { (c. 106b) M. Cino essendo a Perugia. }
$$

20. Perchz uoi state forsi anchor pensiuo, 2 nuoue, che c., 3 delli, 4 mie st., 8 chiara, 10 intento, I 1 uari, 13 El.

$$
\text { (c. 1072) Gherardo da Reggio a M. Cino. }
$$

21. Con sua saetta $d$ 'or percosse amore, 2 merze, 10 respondete, 13 Como potr' io suffrir chessi $m$.

(c. 107b) Risposta di M. Cino et consiglio sopra'l ditto sonetto.

22. Amor che uiene armato a doppio dardo, 3 dell auro, el n., $4 E$ il, 8 Ille tranmutd, 9 receuere, II allei, 14 uuol.

$$
\text { (c. 1082) M. Cino. }
$$

23. Quando ben penso il piccolino spatio, 2 uuole, 4 ueggio, 6 Morti, 7 schuole, 9 Donqua, 10 Amar .... uirtute, 12 delle o. fatte, 13 contrario, 14 Cossi, hauere in salute.

$$
\text { (c. 108b) M. Cino. }
$$

24. Naturalmente ogni animale ha uita, 2 no $s$ 'a., 6 le dil., 10 aduien, alloro, 12 mille anni. Riproduco per intero la $2^{2}$ strole, che doveva essere malconcia già nell' originale:

Amore che innamora altrui per pregio

De uirtù somma che nasce ne l'almo

La qual a dio pareggia

Et quelli che s' addobba del suo fregio

Et stimola et inueggia

Et opera diuerso et uaio

Et affetto nel mondo ne diletto

Che havere el suon de la verace fama

Po chi non la brama

Sta come nel giardin la secca rama. 


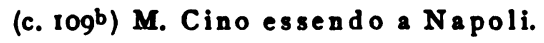

25. Dhe quanda riuedrd il dolce paese, 7 buon, 9 truova, 10 Perho che it $c$. nuova, I pellegrina, 12-1 3 facesti - Del uenir qui non $t$ ' era m. m., 19 Come scimia mi stranno senza lingua, 20 bene a., 23 delli a. uiti et her., 22 inuidia, 23 ualore, se oblia, 25 legiadria, 26 teco sogiunge, 28 transformi, 32 buon, 35 ritien, wol.

(c. IIIa) M. Cino per lo imperadore Henrico di Lucimburgo quando mori.

26. Da poi che la natura ha fine posto, 10 il, 11 intiera, 12 che ho, 14 cemera (sic), 15 in s., 19 quai, 20 che, 21 in, 22 in, 23 ciaschun, 26 in, 27 sommo, 32 alegrezza, 34 iocondo, 39 Muovi, 44 trouarai, 45 Della.

(c. $13^{b}$ ) M. Cino a M. Gherarduccio Garisendi da Bologna.

27. Caro mio Gherarduccio io non ho ueggia, 3 che A., 4 Perho, 5 chaffeggia, 6 uuole, 7 conuien, che, 8 Dell' uno all' a., suole, Io dentro, 13 del.

(c. II $4^{\text {a) }}$ Risp. di M. Gherarduccio.

28. Non pud soir d' amor chi non pareggia, 4 il, 6 el uol., disuole, 7 Perho che amore chel mi signoreggia, 8 Gradisce, 9 Non mercè seruire, 10 buona 1 f feri, 14 uo to vedesti alhor.

\section{(c. $\left.14^{b}\right)$ M. Cino a Dante.}

29. Cercando di trouar lumera in oro, 4 il s., 8 one, 10 fusse, 14 Che ha.

(c. $\left.115^{a}\right)$ Risposta di Dante a M. Cino.

30. Degno ui fa trouare ogni thesoro, 3 il, 4 steccho, 5 trafitto, 6 sospir, 8 uirtu.

(c. $15^{\text {b) }}$ M. Cino a M. Gherarduccio.

31. Amato Gherarduccio, quando i' scriuo, 2 che ad, 4 lungi, 5 mi a., 8 che non, I 1 stincta, 13 dar.

(c. I 16a) Risposta di M. Gherarduccio.

32. Dolce d' amor amico i'vi descrivo, 2 piacemi, 4 scivo, 10 membrandoni, 13 disianza, 14 d' ella purum (?) cinta.

(c. I16b) M. Cino a M. Gherarduccio sopra la detta materia.

33. Come li saggi di Neron crudele, 2 il fecer, 7 com, 14 ch' a mar.

(c. II $7^{a}$ ) Risposta di M. Gherarduccio a M. Cino.

34. Poiche'l pianeto ui da fe certana, 2 mastro, 4 in, 7 Perho, 8 conuien, 9 in tal, 10 Domando, 12 Che amor.

(c. $\left.117^{b}\right)$ M. Cino essendo a Pisa.

35. Al mio parer non e chi in Pisa porti, 3 el, 5 colpi, 7 elli, 8 ha, 9 in, 10 egli] lui, 13 cauallieri.

(c. 118a) Risposta di Guelfo Taviani.

36. Molto il tuoi pensier mi paion torti, 3 fin, l' ha, 4 cauallier, 5 nutricassi, 7 amore che $n$ ' han finta, 8 disij, 9 come a, 13 tai la cera, 14 dalla.

(c. $118 \mathrm{~b})$ M. Cino.

37. Alla battaglia ove madonna abbatte, 2 uirtì, 3 addorno, 4 sin, 7 ansi, de, 8 regha, pensier, 10 i c. suoi, II cauallier, 12 ueggiendo, 13 se. 
(c. I19a) Risposta di M. Guelfo.

38. Pensando come $i$ tuoi sermoni adatte, 2 repugnansa, 3 cauallier, 4 unde, 5 Hor manca, 6 reso, 9 infrascha poi che, 12 Fructe, 13 Elle sue soie, 14 contra quei.

(c. I1 $\left.{ }^{b}\right)$ M. Honesto da Bologna a M. Cino.

39. Poscia che in cor l' amorosa radice, 3 dispiatata, ing., 4 condutto, 5 mie, 6 Et edi, 7 dell' a., 8 ciaschuno, 12 El, 13 mixto.

(c. 120a) Riposta di M. Cino a M. Honesto bolognese.

40. Anzi ch' amore ne la mente guidi, 3 conuien, 8 allui me, 9 suggetto e, 12 Donque, 14 conuiene.

(c. $\left.120^{b}\right)$ M. Honesto a M. Cino.

41. Assai son certo che sementa in lidi, 3 chella, 6 trouar, I 1 con d., proscriptione et carte, 12 cognosci, 13 allegresue, 14 ciaschuna.

(c. I2!a) Risposta di M. Cino a M. Honesto.

42. Se mai legiesti $i$ uersi delli Ouidi, 3 isdegnoso, 6 in, I I uertì, 12 del.

$$
\text { (c. 121b) Canzon di M. Cino. }
$$

43. Mille volte richiamo il di mercede, 3 disiosa, 4 se, 6 ogn' altra, 17 che ho, 19 uirtute, 21 quando, 22 quando io, 23 distino, 24 porria, 25 uenessi, 28 che ogni ragione, 29 in manca, 35 et me peggior che, 36 Donque, 37 grido.

$$
\text { (c. } \left.123^{\mathrm{a}}\right) \text { M. Cino. }
$$

44. Lo sottil ladro che ne gli occhi porti, 3 inuola, 5 che a, 6 suspirando, 8 in, morti, 12 et manca.

\section{(124a) Canzone di M. Cino.}

45. Non che in prensentia della uista humana, 3 all' aud., 6 Che ogni, rasembro, 9 conuien, 10 uirtute, suggetta, 14 in, 17 quel hor, 20 sud., 22 fuggite in. allo int., 23 Ahi zoioso, 24 celo, 25 possian, 32 io, 37 conuiegni, 38 zoioso, 4 uuol, 42 il.

(c. $\left.125^{b}\right)$ Zampa Ricciardi sopra la morte di M. Cino.

46. Morto è colui ch'era archa de la leggie, 2 che, 3 murt'è, 4 rhima, 5 Mort' 2, correge, 7 qual, 8 douria ciaschun, 9 somm'a., 10 le st., 13 Anni] Et mi, 14 di, 16 el $P$.

(c. 126a) Sonet to di Manuel Giudeo.

47. Amor non lesse mai l'auemaria, 7 come pianto, 9 gentile, 13 attiene a c. che gli $m$.

(c. 126b) Cecco Angiellieri a Dante.

48. Dante Aleghier, Ceccho tuo seruo e amico, 4 quale, 12 chore, 13 intendo adonque, 14 tu trouare.

(c. $\left.127^{a}\right)$ Il detto a Dante sospradetto.

49. Dante Aleghier $s$ ia son bon begolardo, 4-5 $s^{\prime} i$ cimo'l panno et tu ui freghi'l cardo, $-S$ 'io mordo'l grasso et tu ne succi'l lardo, $6 S^{\prime} i$ ', $7 S^{\prime} i$ 'sbocco; 9 lodato, 10 nui, 12 uuo, 13 ch' io, 14 pungiglione.

(c. $\left.127^{b}\right)$ M. Chane della Schala a Bologua.

50. Ghelphi, el gran prence nobil de Stericcho, 2 che ha, 3 allontanar, In 
fondo alla c. $127^{\mathrm{b}}$ del cod. Amadei si legge: Albertus Austriae et imperator.

$$
\text { (c. 128a) M. Cino. }
$$

51. Degno son io di morte, 6 sapesti, ne usci, 15 so, 22 ancide, 30 conuien, 33 Io, 34 ben, 35-36 Dunque il mio folleggiare - Piacciaui perdonare, 37 per, uincaui, 4I uenghiare] uemiare, poi corretto sul rigo: ch' ei puì gastigare.

52.

$$
\text { (c. 1302) M. Cino. }
$$

Fior di virtù si è gentil coraggio

Et fructo di uirtu si è honore

Et uaso di uirtu si è ualore

Et nome di uirtu si è huom saggio.

Specchio di uirtu non uede oltraggio

Et uiso di uirtu chiaro colore

Et amor di uirtu buon seruidore

Et dono di uirtu gentil lignaggio

Et luogo di uirtu è conoscenza

Et sadia di uirtu amor reale

Et braccia di uirtu bella accoglienza

Opera di uirtu esser leale

Podere di uirtu è sofferenza

Tutta uirtu è render ben per male.

$$
\text { (c. } 130^{\mathrm{b}} \text { ) M. Cino. }
$$

53. Io son si vago della bella luce, 3 dou' 'e', 7 uirtì, 9 Il q., tutto pien, 11 dopo lo, 12 dol.

$$
\text { (c. 13obis) M. Cino. }
$$

54 .

Io guardo per li prati ogni fior biancho

Per rimenbranza di quel che mi face

Si uago di sospir ch' io ne chieggio ancho

Et mi rimembra della biancha parte

Che fa col uerde brun la bella taglia

La qual vestio amore

Nel tempo che guardando ueno et marte

Con quella sua saetta piu taglia

Mi die per mezzo il core

Et quando lauro mone il biancho fiore

Rimembro de begliocchi il dolce biancho

Per cui lo mio desir mai non fie stancho.

55.

$$
\text { (c. 13 }{ }^{\mathrm{b}} \text { ) M. Cino. }
$$

Nouelle non di ueritate ignude

Quant' esser puo lontane sian da giocho

Desio sauer si chio non trouo locho

De la biltà che pel dolor si chiude.

Accio ti prego metti tua uirtude

Pensando che entrerai parte in focho

Ma svariato $m$ ' ha forse non pocho

La noua usanza delle genti crude. 
Si che a me lasso al tuo penser non uolte Dero $m$ ' obrij che memoria non perde Se non quel che non guarda spesse uolte.

Ma se del tutto anchor non si disperde

Mandarai a dir merze a chi amo molte

Come si dea mutare il scuro in uerde.

$$
\text { (c. 132a) M. Cino. }
$$

56. Oime ch' io ueggio per entro un pensero, 4 tanto $\grave{e}, 8$ Quando dauanti si duol per lo uero.

$$
\text { (c. } 13^{\mathrm{b}} \text { ) M. Cin o. }
$$

57. Quando potrd io dir dolce mio dio, 2 uirtute, 3 hai, in, 4 Però, com ' 5 Ueder, 8 scuritate, 12 Io m' empio tutto, 14 destringi, 15 Della, 16 In creschati, 18 to 'ntelletto, 19 del, 24 obedir a li angelli. Manca la strofe III e si legge in basso: "manca una stanza vedi a carte 148."

$$
\text { (c. } 133^{b} \text { ) Del Petrarcha. }
$$

58.

Quella girlanda che la bella fronte

Cingeua di color fra perle et grana

Senuccio mio parueti cosa humana

$O$ angeli che forme al mondo aggiunte.

Uedestu gli atti et quelle chiome conte

Chel mio cor morde in un lasso et risana

Uedesti tu il piacer che $m$ ' allontana

D' ogni uile pensiero ch' al cor mi monte.

Odiste il suon delle dolci parole

Mirasti tu l' andar soave altiero

Dietr' al quale ho suiato $i$ pensier mei.

Soffriste il sguardo inuidioso al sole

Dunque sai per cui uiuo moro et spero

Et non so dimandar quel ch' io uorrei.

(c. 1342) Del Petrarcha.

59. Sostenne con le spalle Hercole il cielo. Manca nel casanatense.

\section{Appendice.}

Sonetti nella lezione del cod. Galvani.'

I.

Omo saccente: è da Maestro saggio

De interrogar per apparar savere

Ond' io mi movo a voi si come a maggio

Dottor che siete per ragion cernere.

S' amor discende per gentil corraggio

$O$ di che nasce o se vien per piacere

1 Riporto questo saggio da una descrizione frammentaria del cod. Galvani dovuta allo stesso co. G. Galvani e conservata, come ho detto, tra le sue carte nell' Autografoteca Campori (Bibl. estense). 
O s' egli ha or se forza o signoraggio

E possa quanto più se ne puó avere.

E prego voi si come più pregiato

Signor che di scienzia $\mathrm{m}$ ' insegnate

D' esto dimando, sire, ch' io vi chero

$\mathrm{Ch}^{\prime}$ io non sono da me tanto insegnato

Che ciò possa saver per veritate,

Ond' io mi torni al dritto sentiero.

II.

Ser Mula, tu ti credi senno avere

Tanto che porta virtù l' Elitropia

Che di cosa comune è fatto propria

Ma non come ti pensi al mio parere.

Nostra ragione pur ci conven cernere

$E$ dice facciam prego a donna inopia

Che venga tosto si che n' aggiam copia

Di poterla toccar non che vedere.

$\mathrm{Ma}$ ben crebbe rimedio al nostro inganno

$\mathrm{Ch}^{\prime}$ ella sposasse quella pulcelletta

Celatamente si che tutti 'l sanno.

E sappiam ben che tu trovasti stretta

Si come quella ch' era nel sest' anno

Rilegati Ser Mula cotal vetta.

III.

Bindo Bonichi.

Mostraci '1 mondo prode e dacci danno

Promettici allegrezza e dacci pena

La cosa turba ci dà per serena

Nudo si trova chi su veste panno

Quei che sono in tormento il ver ne sanno

Legati stanno con la sua catena

Com 'elli è falso e traditor si mena

Ed è senza pietà crudel (?) tiranno

Ma tu che sai chi fa di sua brigata

Dame per dolce tal bevanda a bere

Ch' a rivender cara fia la derrata.

Ed ogni cosa ha messo a non calere

La non discreta turba dispietata

Che 'l ver non segue, ma' 1 folle parere.

Questo consiglio dà Bindo Bonichi,

Messer Bennuccio, colli saggi antichi. 
IV.

Benuccio Salimbeni.

A fine di riposo ho sempre affanno

$E$ zappo in acqua e semino su rena

$E$ la speranza mi lusinga e mena

$D^{\prime}$ oggi in domane e così passo l' anno.

$E$ son canuto sotto questo inganno

Senza poter raccoglier un di lena,

Ma la speranza paura raffrena

Vedendo come gl' annì se ne vanno.

E temo ch' io non compia mai giornata

Senza potermi ponere a sedere

E terza ora e nona è già sonata.

Poi viene 'l vespro e vorrei volere

Da capo fare una bella levata;

Ouesto vorrei, ma non ho più potere.

V.

Manoel Giudeo.

Io che trassi le lagrime del fondo

Dell 'abisso del cor che 'n su le envea

Piango che 'l fuoco del duolo $m$ 'ardea

Se non fossero le lagrime in che abbondo.

Che la lor pioua ha mortal profondo

Ardor che del mio mal fuor me traea

Per non morir, per tener altra vea

A percoter sto forte e non ha fondo.

E ben po' pianger Christiano o Iudeo

$E$ ciascun uedere en tristo scanno

Pianto perpetual m' a fatto reo

Perch' $i$ ' $m$ ' accorgo che quel fu 'l malanno

Sconfortomi, bench' i' ueggio che Deo

Per inuidia $-\ldots-\ldots$

1 Così nel manoscritto del Galvani (Autografoteca Campori).

Givlio Bertoni. 